\title{
A Sensitivity Mapping Technique for Tensile Force and Case Depth Characterization Based on Magnetic Minor Hysteresis Loops
}

\author{
Cunfu He, Wenqiao Dou, Xiucheng Liu* ${ }^{*}$, Meng Yang and Ruifang Zhang
}

\begin{abstract}
In the nondestructive testing and evaluation area, magnetic major hysteresis loop measurement technology are widely applied for ferromagnetic material evaluation. However the characterization ability of major hysteresis loop measurement technology greatly varies as the evaluated target properties. To solve this limitation, magnetic minor hysteresis loops, which reflect the responses of ferromagnetic material magnetization in a systematic way, is recommend. Inspired by plenty of information carried by the minor loops, the sensitivity mapping technique was developed to achieve the highest sensitivity of minor-loop parameters to the nondestructively evaluated targets. In this study, for the first time, the sensitivity mapping technique is used to measure the tensile force in a steel strand and evaluate the effective case depth in induction-hardened steel rods. The method and procedures for the sensitivity mapping technique are given before experimental detection. The obtained experimental results indicate that the linear correlation between the induced voltage (or the magnetic induction intensity) and the tensile force (or effective case depth) exists at most of the locations in the cluster of minor loops. The obtained sensitivity maps can be used to optimize the applied magnetic field (or excitation current) and the analyzed locations at the minor loops for achieving the highest sensitivity. For the purpose of tensile force measurement, it is suggested that the strand should be firstly magnetized to the near-saturation state and then restored to the remanent state. In this way, the highest sensitivity is obtained as about $15.26 \mathrm{mV} / \mathrm{kN}$. As for the induction-hardened steel rods, the highest sensitivity of magnetic induction intensity to the effective case depth occurs under low magnetic field conditions and the absolute value of the highest sensitivity is about $0.1110 \mathrm{~T} / \mathrm{mm}$. This indicates that if the highest sensitivity is required in the case depth evaluation, the induction-hardened steel rods are only required to be weakly magnetized. The proposed sensitivity mapping technique shows the good performance in the high-sensitivity evaluation of tensile force and case depth in ferromagnetic materials and its application scope can be extended to other nondestructive detection fields.
\end{abstract}

Keywords: Sensitivity mapping technique, Magnetic minor hysteresis loop, Tensile force, Case depth

\section{Introduction}

The measurement of the magnetic hysteresis curves is a promising pathway to achieve the nondestructive evaluation (NDE) of the properties of ferromagnetic materials [1]. Due to the action of periodic magnetic field, the magnetic domains interact with the micro-structures and the magnetization response of the material can be well

*Correspondence: xiuchliu@bjut.edu.cn

Faculty of Materials and Manufacturing, Beijing University of Technology, Beijing 100124, China illustrated by the trajectory of the hysteresis loops [2]. Traditional feature parameters (such as coercive force and saturation and residual magnetic induction intensity) extracted from specific locations of the major loop are important indicators for characterizing both the microstructural changes caused by heat treatment or plasticity and the residual or working stress state of ferromagnetic components $[3,4]$.

The traditional feature parameters are easily extracted and can well reflect the magnetic property of ferromagnetic materials. However, from the perspective of NDE, 
the sensitivity of the traditional feature parameters to the target properties is not necessarily the highest. In addition, due to the diversity of target properties in NDE applications, the universality of the frequently used major loop measurement method is debatable [5]. To clarify the two questions, the systematic measurement methods of magnetic minor hysteresis loops are suggested for NDE applications. Multiple candidate parameters are extracted from the measured cluster of minor loops for the characterization of target properties and the performances of the extracted candidate parameters are discussed in the space made of minor loops $[6,7]$.

Takahashi et al.[8, 9] and Kobayashi et al. [10, 11] proposed the magnetic hysteresis scaling method with the large families of minor loops and found several scaling power laws between minor-loop parameters and the properties of microstructures. More importantly, the coefficients of the power laws were proportional to mechanical properties including ductile-brittle transition temperature, hardness and yield stress. Tomáš et al. [12, 13] and Vértesy et al. [14] developed an innovative magnetic adaptive testing technique for evaluating the plastic strain and ductile-brittle transition temperature, which were sensitive indicators of material degradation. In the magnetic adaptive testing technique, the cluster of minor hysteresis loops is measured for each of the degraded samples and then the differential-permeability-related parameters are calculated at any point on any minor loop. Consequently, the obtained sensitivity map can help to reflect the material degradation in the most sensitive way.

As a powerful tool for nondestructive evaluation of the properties of ferromagnetic materials, the sensitivity mapping technique (SMT) can be applied in many other NDE fields. Inspired by the previous reports [12-14], in this study the sensitivity mapping technique is first explored for tensile force measurement in a pre-stressed seven-wire steel strand and case depth evaluation in induction-hardened carbon steel rod. It is worth noting that the manifestation form of the measured signals carrying the minor loop information determines the pattern of the sensitivity map. To the authors' knowledge, the manifestation form of measured B-H minor loops have not yet been employed for SMT. In this work, the feasibility of B-H minor loops integrated with SMT will be investigated for high-sensitivity characterization of case depth in induction-hardened carbon steel rod.

Though the SMT can be customized to specific applications, the magnetization conditions corresponding to the highest sensitivity was found to be target properties-dependent by comparing the conclusions obtained from the two investigated cases in this study. The presented work shows that the linear correlation between the minor-loop parameters (induced voltage or magnetic induction intensity) and the target properties (tensile force or case depth) exists at most locations along the minor loops. The strength of the applied magnetic field should be as high as possible to allow the strand to reach its near-saturation state. Then the highest sensitivity of induced voltage to the variation of tensile force can be achieved at the minor loop location, where the rate of change in the magnetic induction intensity is the highest. However, it is suggested that the highest sensitivity can be obtained within the weakly magnetized samples and that the minor-loop location corresponding to the highest sensitivity has the maximum magnetic induction intensity.

The rest of the paper is organized as follows. In Section 2 , the procedures for the derivation of sensitivity map are introduced. The sample preparation and the experimental set-up for measuring minor loops in a tensioned steel strand and induction-hardened steel rods are described in Section 3. In Section 4, the characters of the obtained sensitivity map are analyzed and the quantitative characterization results of target properties with the minor-loop parameters are discussed in detail. Finally, the conclusions are drawn in Section 5.

\section{Sensitivity Mapping Technique}

The cluster of the minor loops demonstrates the magnetization responses of the tested samples which are subjected to an alternating magnetic field with the progressively increasing strength $[15,16]$. For the cylindrical samples in this study, the solenoid coils-based sensor is employed to measure the minor hysteresis loops. Amplitude-modulated sinusoidal current $I_{E}(t)$ shown in Figure 1a is fed into the primary coil to provide an alternating magnetic field $H(t)$. The induced voltage $U_{o}(t)$ in the secondary coil can be converted into magnetic induction intensity $B(t)$. The measured results can be displayed in the form of $B-H$ chart to obtain the cluster of minor hysteresis loops (Figure $1 \mathrm{~b}$ ), or in the form of $U_{o}-I_{E}$ chart to form the quadrilateral trajectories (Figure 1c). The results in Figure 1b and Figure 1c can be used to represent the magnetization process of materials even though they are in different expression forms. For the convenience of description, both results in Figure 1b and Figure 1c are referred to as measurement results of minor loops in this study.

Due to the symmetry of the signal trajectories, the measured minor loops can be analyzed in a half-space of the applied magnetic field or the current. More precisely, only the ascending (or descending) branches of all the measured minor loops or the quadrilateral trajectories will be intercepted from the results for the following matrixing operations. Here, a single ascending branch of a minor loop in $U_{o}-I_{E}$ chart is taken as an example to illustrate the procedure of sensitivity 

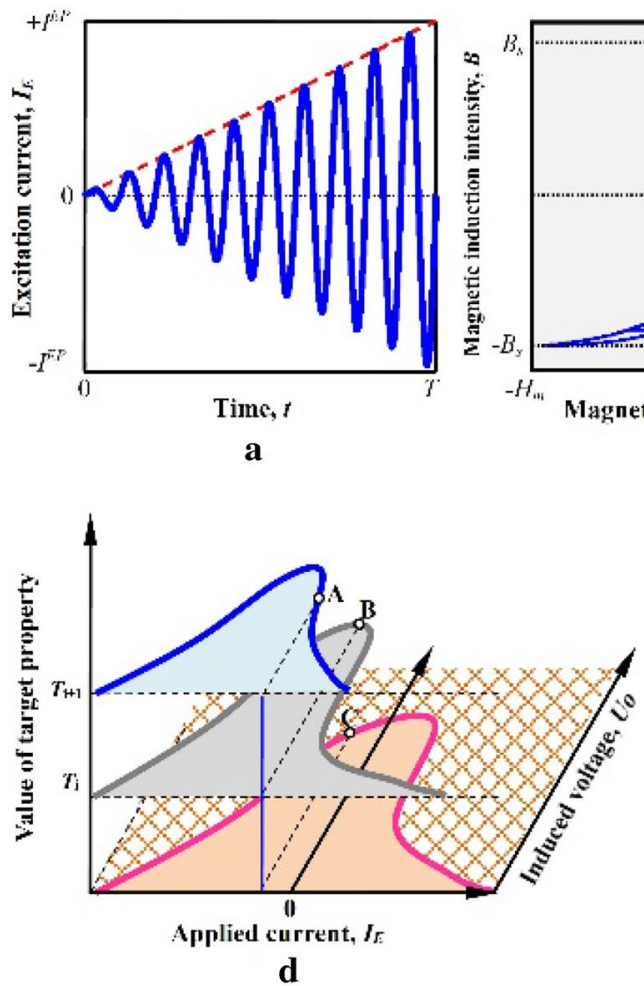

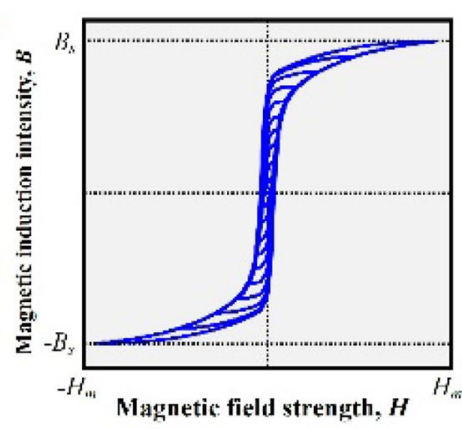

b

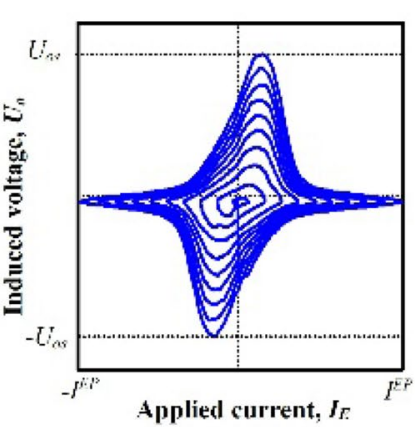

c

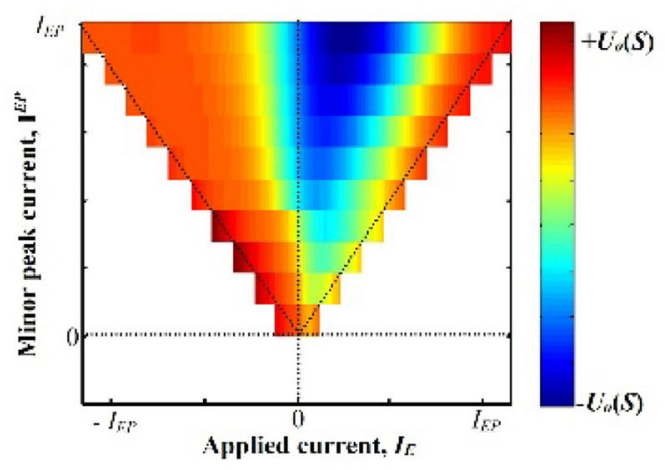

e

Figure 1 Formation process of the sensitivity map: a When the tested material is placed inside a primary coil fed with an amplitude-modulated sinusoidal current, $\mathbf{b}$ Magnetization responses of the material can be characterized by the cluster of minor hysteresis loopsor, c Trajectories of the induced voltage varying with excitation current. $\mathbf{d}$ Diagram of the interpolated minor loops, and $\mathbf{e}$ Map representing the matrix of induced voltage $U_{0}$ or sensitivity $S$

mapping techniques. The measurement of minor loops is conducted on each specimen and then the relationship between the feature parameter (the induced voltage amplitude) and the value of target property $(T)$ is investigated with the results shown in Figure 1d.

Unavoidable noise causes minor differences among the repeatedly measured applied current. Therefore, it is difficult to achieve the uniform amplitude sampling of the applied current during the tests [17]. To provide the common coordinate of current $I_{E}$ for the analysis of minor loops, continuous uniform interpolation operation of the measured applied current is performed in MATLAB software. The amplitude of each cycle of the excitation current is represented by a one-dimensional matrix of $M$ elements. Therefore, the current of $N$ cycles can be illustrated by a two-dimensional matrix of $N \times M$ elements as follows: where $I_{i j}^{E P}(i=1,2, \ldots, N ; j=1,2, \ldots, M)$ is the peak amplitude of each cycle of the current (referred to as minor peak current). The induced voltage of $U_{o}$ is subjected to similar interpolation operation to obtain a twodimensional matrix of $\left(U_{i j}^{o}\right)_{N \times M}$. The matrix of $\boldsymbol{I}_{E}$ acts as a datum map and at each pixel (or element of $I_{i j}^{E P}$ ) the correlation between $U_{i j}^{o}$ and the target property $(\boldsymbol{T})$ is evaluated by simple linear curve fitting tool,

$$
U_{i j}^{o}=\boldsymbol{a}_{i j} \boldsymbol{T}+\boldsymbol{b}_{i j}
$$

where $\boldsymbol{a}_{i j}$ and $\boldsymbol{b}_{i j}$ are the matrix of the slope and intercept of the fitting straight lines, respectively. It should be noted that the approximately linear dependency of $U_{i j}^{o}$ on the property of $\boldsymbol{T}$ is not adequate for the entire space of $\boldsymbol{I}_{E}$. The coefficient of determination, $R^{2}$, is employed

$$
\boldsymbol{I}_{E}=\left[\begin{array}{ccccccccc}
-I_{11}^{E P} & \ldots & \cdots & \ldots & \ldots & \ldots & \ldots & \ldots & +I_{1 M}^{E P} \\
0 & -I_{2(j)}^{E P} & \cdots & \ldots & \ldots & \ldots & \ldots & \ldots+I_{2(M-j+1)}^{E P} & 0 \\
0 & 0 & \ddots & \ldots & I_{i j}^{E P} & \ldots & . & 0 & 0 \\
0 & 0 & 0 & -I_{N(j)}^{E P} & \cdots & +I_{N(M-j+1)}^{E P} & 0 & 0 & 0
\end{array}\right]_{N \times M},
$$


to evaluate the linear curve fitting result obtained at each pixel in the map. If the sum of squares due to error (SSE) and the total sum of squares (SST) of the fitting are known, the coefficient of determination can be expressed as $R^{2}=1-\mathrm{SSE} / \mathrm{SST}$. The value of $R^{2}$ indicates the goodness of fit of the linear equation and ranges from zero to one. The greater the value of $R^{2}$, the higher the goodness of fit. Only the dataset corresponding to the pixels with a value of $R^{2}$ higher than the threshold value is selected for linear curve fitting. The obtained matrix of slope $\boldsymbol{a}_{i j}$ can be expressed in the form of an inverted isosceles triangular map, as shown in Figure 1e. The slope of well fitted straight line can be treated as the sensitivity of dependent variable to the independent variable. Therefore, the map shown in Figure 1e is adequate for analyzing the sensitivity of the minor loop parameters to the target NDE property.

The final result of the sensitivity map highly depends on the excitation current, the selected datum map, the analyzed minor-loop parameter and the target property for characterization. For instance, the maximum peak amplitude and the cycle number of the excitation current determine the material magnetization region and the vertical resolution of the datum map, respectively. The results of measured tangential magnetic field can also be applied to form a new datum map, thus leading to coordinate scaling of the final sensitivity map. In addition, the selection of the minor-loop parameters to be analyzed, such as the induced voltage, the magnetic induction intensity and the permeability, causes scaling in the sensitivity values. Moreover, even if the procedures of the experiment and the formation of sensitivity map are fixed, the high-sensitivity region in the map varies with the target property. The above flexibilities in the formation of sensitivity map enable the sensitivity mapping technique (SMT) to be a powerful tool for the nondestructive evaluation of target property. In the subsequent experiments in Section 3, the SMT illustrated in Figure 1 is alternatively applied to characterize the tensile force in a seven-wire steel strand and the case depth in inductionhardened carbon steel rods.

\section{Experiments}

\subsection{Tensile Force Measurements in Steel Strands}

The magnetization responses of a seven-wire steel strand, which is commonly used in pre-stressed structures, are measured on the experimental set-up shown in Figure 2. Six galvanized high-carbon steel wires are twisted around a central steel rod to form a strand with a nominal diameter of about $17.8 \mathrm{~mm}$. A custom-developed stretching system is employed to apply tensile force to the strand. As shown in Figure 2, a hydraulic cylinder is used to

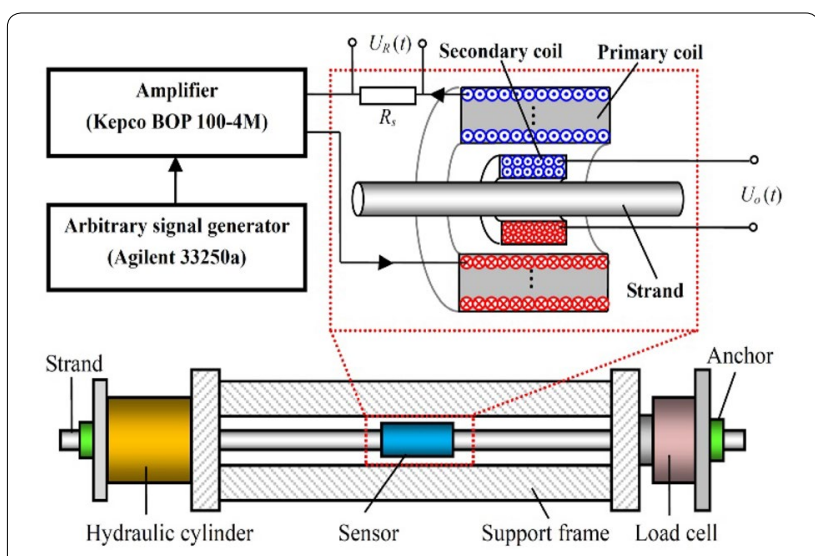

Figure 2 Experimental set-up for measuring the magnetization responses of a seven-wire steel strand under the action of different tensile forces

apply an axial load on the tested strand, whose both ends are anchored onto the stretching system, and a load cell is attached to the support frame to record the value of the applied tensile force [18].

The sensor for measuring the minor hysteresis loops of the strand deploys a primary coil and a secondary coil. The primary coil is made by winding four layers of enameled copper wires with a diameter of $0.25 \mathrm{~mm}$ onto a plastic bobbin. The primary coil has a length of $80 \mathrm{~mm}$ and its total number of coil turns is about 1280 . The dimension of the secondary coil is about $15 \mathrm{~mm}$ in length and $19 \mathrm{~mm}$ in inner diameter. The total number of coil turns of the secondary coil is about $N_{\mathrm{c}}=444$.

A linear amplitude-modulated sinusoidal current with a frequency of $2 \mathrm{~Hz}$ is generated by an Agilent 33250a function generator. The current signal has twenty cycles and is fed into the primary coil after being amplified by a KEPCO BOP-400L power supplier to yield a maximum peak-to-peak amplitude of 4.0 A. A resistor with a resistance of $1 \Omega$ is connected to the primary coil in series to acquire the voltage of $U_{R}(t)$ for excitation current sampling. The signal of $U_{R}(t)$ and the voltage induced in the secondary coil $U_{o}(t)$ are synchronously acquired by a Tektronix 4490b digital oscilloscope with a sampling rate of $500 \mathrm{~S} / \mathrm{s}$.

It is worth noting that the measured signals of $U_{R}(t)$ and $U_{o}(t)$ are related with the sizes and winding of both primary and secondary coils $[19,20]$. For the tested cylindrical samples with a given diameter, one can design the configuration of the coil sensor according to actual needs. Though change of coil may shift the shape of the measured voltage signals, the effectiveness of the proposed sensitivity mapping technique remains. The waveforms of the signals of $U_{R}(t)$ and $U_{o}(t)$ obtained from a free strand (tensile force $F=0 \mathrm{kN}$ ) is plotted in Figure 3a. 


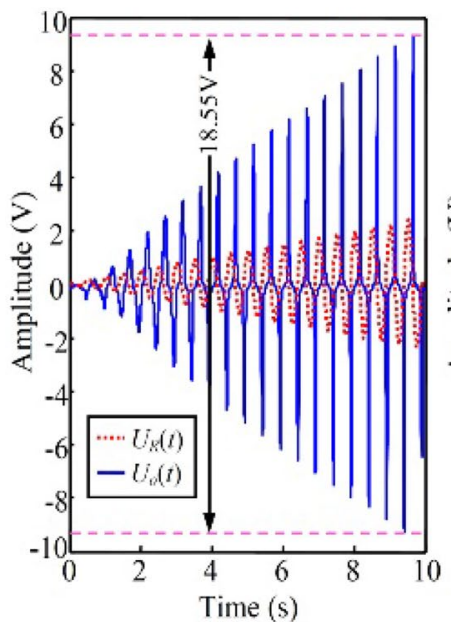

a

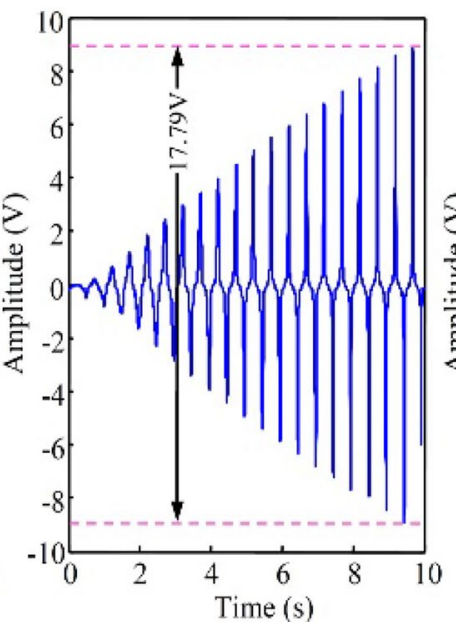

b

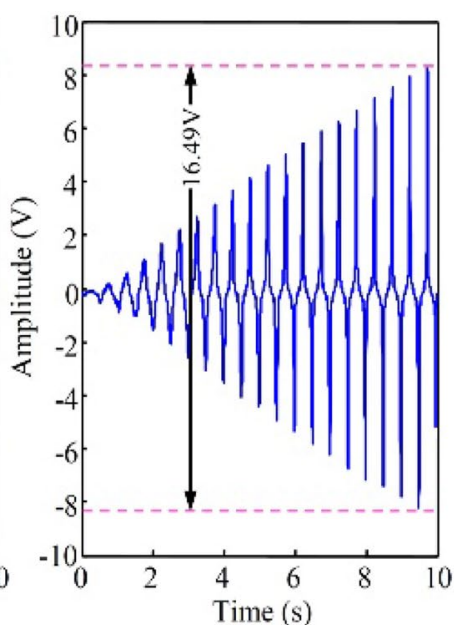

c

Figure 3 Induced voltage signal in the secondary coil when the applied tensile force is about: a $0 \mathrm{kN}, \mathbf{b} 39.2 \mathrm{kN}$ and c $78.4 \mathrm{kN}$.

The maximum peak-to-peak amplitude of the induced voltage $U_{\mathrm{os}}$ is around $18.55 \mathrm{~V}$. The tensile force of the strand successively increases from $0 \mathrm{kN}$ to $98.0 \mathrm{kN}$ with a step of $19.6 \mathrm{kN}$, whereas the value of $U_{\text {os }}$ demonstrates the monotonously decreasing behavior, as confirmed from the results obtained at the tensile force of $F=39.2$ $\mathrm{kN}$ (Figure $3 \mathrm{~b}$ ) and $F=78.4 \mathrm{kN}$ (Figure 3c).

\subsection{Case Depth in Medium Steel Rod}

Medium carbon steel $(0.48 \%$ of $\mathrm{C}, 0.18 \%$ of $\mathrm{Si}, 0.56 \%$ of $\mathrm{Mn}, 0.052 \%$ of $\mathrm{Cr}, 0.013 \%$ of $\mathrm{Cu}$, and $\mathrm{Fe}$ ) is selected as raw materials for heat treatment. Five steel rod samples were prepared and then four of the five samples were respectively heated to $840{ }^{\circ} \mathrm{C}$ for $1800 \mathrm{~s}, 1500 \mathrm{~s}, 1300 \mathrm{~s}$, and $700 \mathrm{~s}$ in a high-frequency induction coil to achieve different case depths, followed by cooling in water. The oxide skin on the sample surface was removed and all the five samples had the same diameter of $22.5 \mathrm{~mm}$ and the same length of $200 \mathrm{~mm}$. Before each experimental test, sample demagnetization was conducted with a commercial demagnetization device. After the demagnetization process, the surface magnetic field strength of the steel rods was measured with a Gauss meter to make sure that the surface magnetic field strength was lower than $1 \mathrm{Gs}$.

The sensor for measuring the minor loops of the steel rod sample is shown in Figure 4a. Primary and secondary coils are embedded into the pure iron shell. The inner diameter and the length of the primary coil are about $44 \mathrm{~mm}$ and $100 \mathrm{~mm}$, respectively. The secondary coil has an inner diameter of $26.5 \mathrm{~mm}$ and a length of $20 \mathrm{~mm}$. The total number of the coil turns in the primary coil and secondary coil is about 814 and 444 , respectively. Two U-shaped pure iron yokes were mounted onto the surface of the steel rods to minimize the flux leakage magnetic

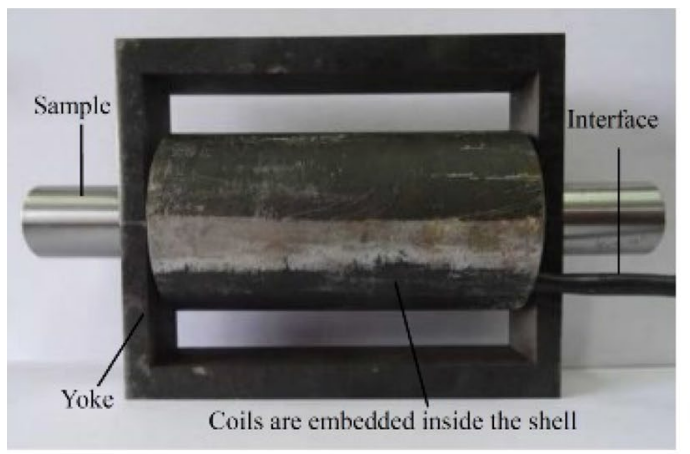

a

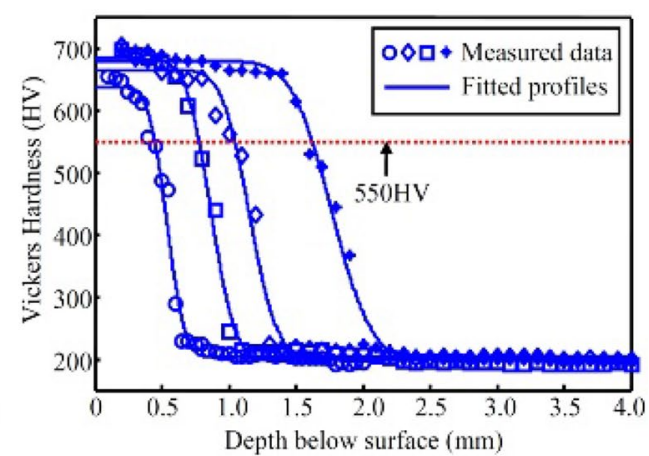

b

Figure 4 a Picture of the sensor for measuring the minor hysteresis loops of induction-hardened steel rods and $\mathbf{b}$ micro-hardness test results (symbols) and the fitted curves of hardness-depth profile (solid lines) 
field during the sample magnetization process. A Hall sensor (UGN3503U, Honeywell) was placed near the secondary coil to measure the tangential magnetic field in the rod surface.

Micro-hardness tests were performed on the well prepared samples that were cut from the middle length of the steel rods to determine the case depth in the samples after the measurement of minor loops. The detailed procedure of micro-hardness tests and the curve fitting operation applied to the measured data of Vickers hardness in the depth direction shown in Figure $4 \mathrm{~b}$ with a complementary error function can be found in a recent report [21]. In accordance with the ISO 2639 International Standard, the corresponding depth at the Vickers hardness of $550 \mathrm{HV}$ is treated as the effective case depth, $d_{\text {eff }}$. The estimated values of $d_{\text {eff }}$ in the four quenched samples are about $0.45 \mathrm{~mm}, 0.78 \mathrm{~mm}, 1.05 \mathrm{~mm}$, and 1.63 $\mathrm{mm}$, respectively.

Similar measurement devices shown in Figure 2 are employed to measure the minor loops of induction-hardened steel rod samples. An amplitude-modulated twentycycle sinusoidal current with a frequency of $2 \mathrm{~Hz}$ is fed into the primary coil to yield the magnetic field for sample magnetization. To obtain good measurement results of minor loops, the sinusoidal excitation current with a certain peak-to-peak amplitude lasts for two cycles so that a total of ten minor loops can be measured during the magnetization period. The typical tangential magnetic field $H(t)$, measured by the Hall sensor, and the voltage signal induced in the secondary coil $U_{o}(t)$ are plotted in Figure 5a and Figure 5b, respectively.
The magnetic induction intensity $B(t)$ can be estimated as,

$$
B(t)=\int_{0}^{T} \frac{U_{o}(t)}{N_{c} A_{s}} \mathrm{~d} t+\mu_{0}\left(1-\frac{A_{m}}{A_{s}}\right) H(t),
$$

where $\mu_{0}$ is the permeability of air, $N_{c}$ is the coil turns of the secondary coil; $A_{s}$ and $A_{m}$ represent the crosssectional area of the tested rod and the secondary coil, respectively.

The trajectories of the $B(t)$ varying with $H(t)$ are the minor hysteresis loops, as shown in Figure $5 \mathrm{c}$. The values of the apparent parameters of measured minor loops, such as coercive force and residual magnetic induction intensity, are not consistent with the intrinsic values of the tested materials because the sensor configuration and its operational parameters used in this study are not the same to those used for measuring the intrinsic minor hysteresis loops. Nevertheless, from the perspective of NDE applications, the results in Figure $5 \mathrm{c}$ are valid for case depth characterization. With the successive increase in case depth, a downward trend in the apparent residual magnetic induction intensity can be observed from the results in Figure 5c, whereas the apparent coercive force slightly increases.

\section{Results and Discussion}

To construct the sensitivity map for tensile force measurements in steel strand, the original signals shown in Figure 3 are smoothed according to moving average method with the span of 11 and then redrawn in the $U_{o}-I_{E}$ chart (Figure 6). For the cluster of minor loops

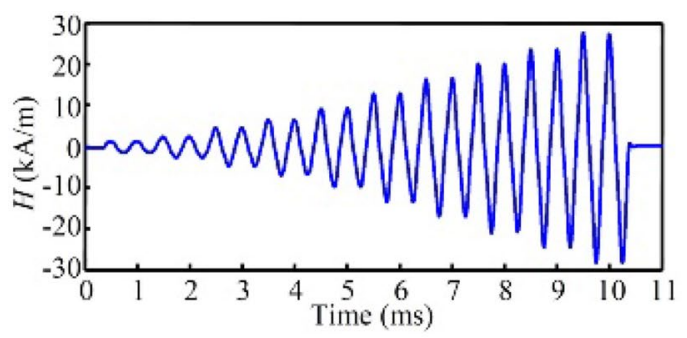

a

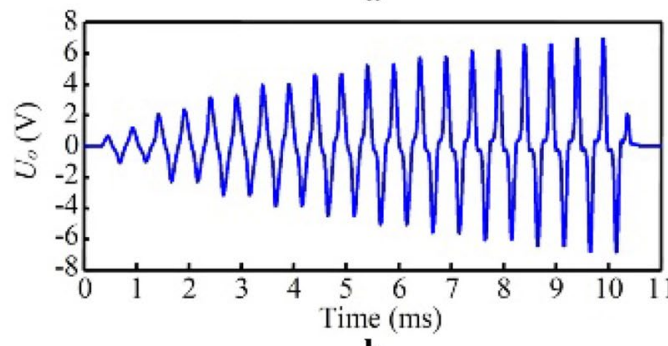

b

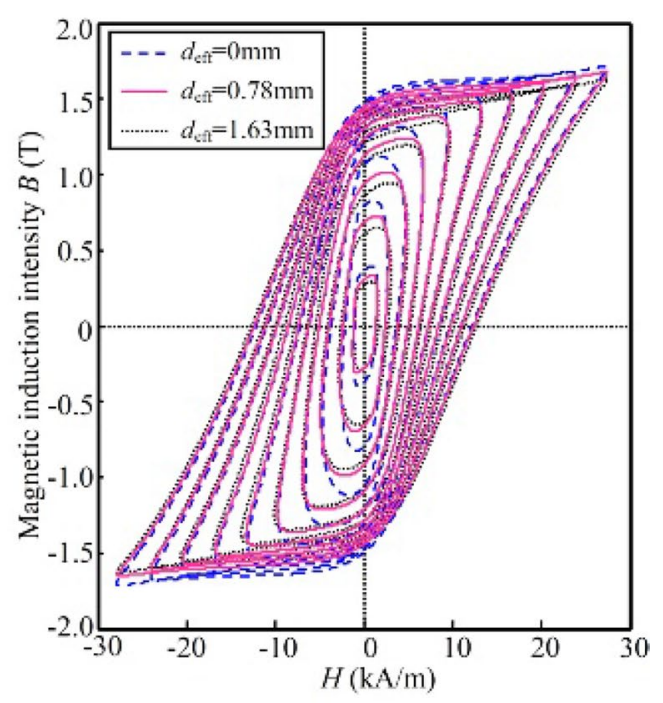

Figure $\mathbf{5}$ a Typical waveforms of the measured tangential magnetic field, $\mathbf{b}$ Induced voltages in the secondary coil, and $\mathbf{c}$ Minor hysteresis loops obtained from the rods with different case depths 


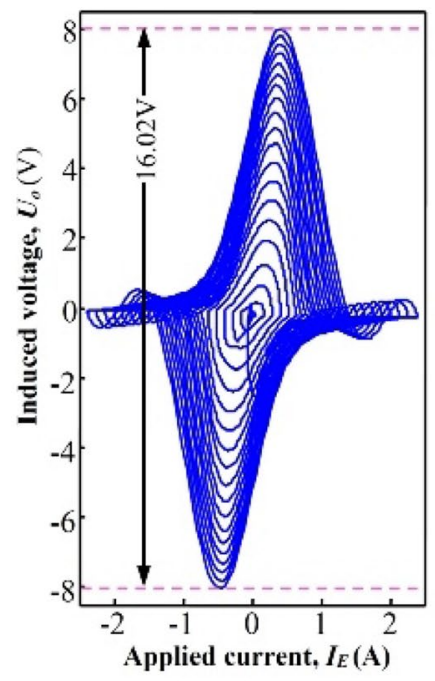

$\mathbf{a}$

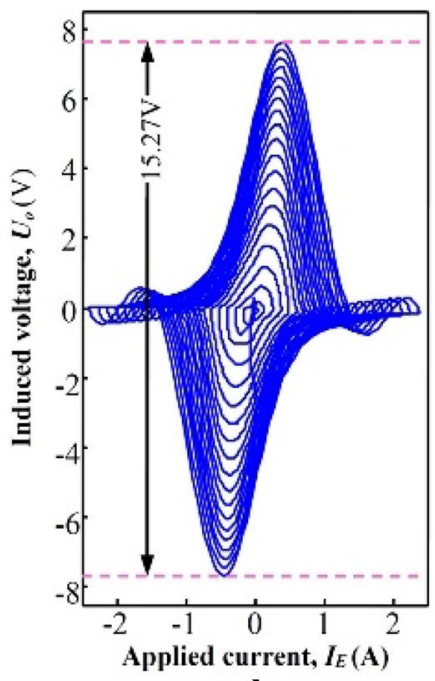

b

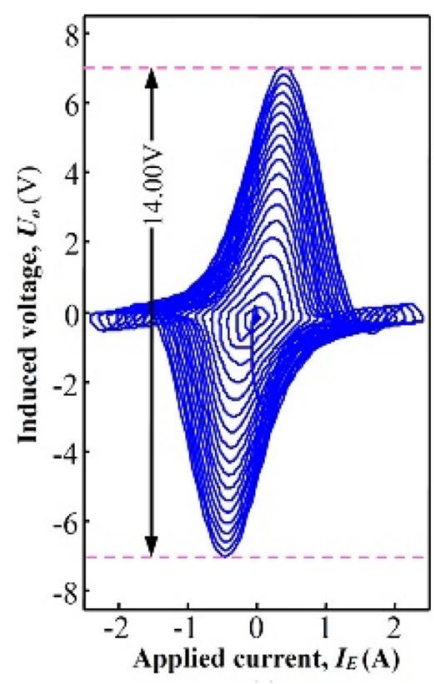

c

Figure $\mathbf{6}$ Trajectories of the induced voltage varying with the applied current when the tensile force is about a 0 kN, b $39.2 \mathrm{kN}$ and $\mathbf{c} 78.4 \mathrm{kN}$

obtained under a certain tensile force, the ascending branches are extracted and the map of induced voltage is derived according to the datum map construction procedures, as illustrated in Section 2. Uniform interpolation is required to the measured waveform of current $I_{E}$ in the process of datum matrix construction. The number of uniform interpolation points determines the column number of the datum matrix. The basic guide lines for the selection of column number is that the interpolated waveform should be consistent with the original waveform as much as possible. In this study, a total of 100 data points are acquired per magnetization cycle and the number of elements per column in the datum matrix of applied current $I_{E}$ is increased to 400 by applying the uniform interpolation operation in the current amplitude range of $\pm 2.39 \mathrm{~A}$. Therefore, the size of the datum matrix is $20 \times 400$.

When the tensile force applied to the strand is $0 \mathrm{kN}$, the $U_{\mathrm{o}}$ map is shown in Figure 7a. To highlight the tensileforce-induced variation in the $U_{o}$ map, the matrix of $U_{o}$ corresponding to free strand case is subtracted from the $U_{o}$ matrix obtained under the tensile force of $39.2 \mathrm{kN}$ and $78.4 \mathrm{kN}$ and corresponding $\Delta U_{o}$ maps are respectively shown in Figure 7b and Figure 7c.

Linear curve fitting operation is applied to the data of tensile force and the induced voltage at each element in the datum matrix and the map of the coefficient of determination $R^{2}$ is plotted in Figure $7 \mathrm{~d}$. The color bar indicates the value of $R^{2}$. In the majority of the $R^{2}$ map, the value of $R^{2}$ is higher than 0.8 , indicating the good linear dependency of $U_{o}$ on the tensile force. However, the value of $R^{2}$ is lower than 0.5 when it comes to the narrow regions with an applied current between $-0.25 \mathrm{~A}$ and $0.50 \mathrm{~A}$ or the regions near the two sides of the triangular map. The $R^{2}$ map can help to rapidly distinguish the regions of interest, in which the measured data can be well fitted with the predetermined equations. In this study, the regions or the pixels in the datum map with a corresponding value of $R^{2}$ less than 0.8 are abandoned in the subsequent construction of sensitivity map.

As for the results obtained from the induction-hardened steel rods, the maps are derived based on a datum matrix of applied magnetic field $\boldsymbol{H}_{E}$. The matrix of $\boldsymbol{H}_{E}$ has a size of $10 \times 400$ and the magnetic field strength of all the elements varies in the range of $\pm 27.47 \mathrm{kA} / \mathrm{m}$. The ascending branches of the cluster of minor hysteresis loops are used to form the matrix of magnetic induction intensity $\boldsymbol{B}$. Figure 8 a shows the color map of $\boldsymbol{B}$ matrix, which is quite different from the results in Figure 7a. The difference is mainly caused by the selection of minorloop parameters in the derivation of maps. When the ascending branches of minor loops are drawn in the form of $U_{o}-I_{E}$, the loops always have the positive $U_{o}$ and the maximum values of $U_{o}$ appear in the region near the middle line of the isosceles triangular map (see Figure 7a). When it comes to the form in $B-H$ chart, the value of $B$ in the ascending branches of minor loops demonstrates the zero-crossing behavior and the peak values of $B$ are always obtained at the boundaries of the map.

For each location of the datum matrix of $\boldsymbol{H}_{E}$, Eq. (2) is used to fit the data of $B$ and tensile force $F$. The map representing the $R^{2}$ matrix is shown in Figure 8b. Interestingly, along each column of the matrix, the value of $R^{2}$ reaches a minimum value (less than 0.7 ) when the minor 

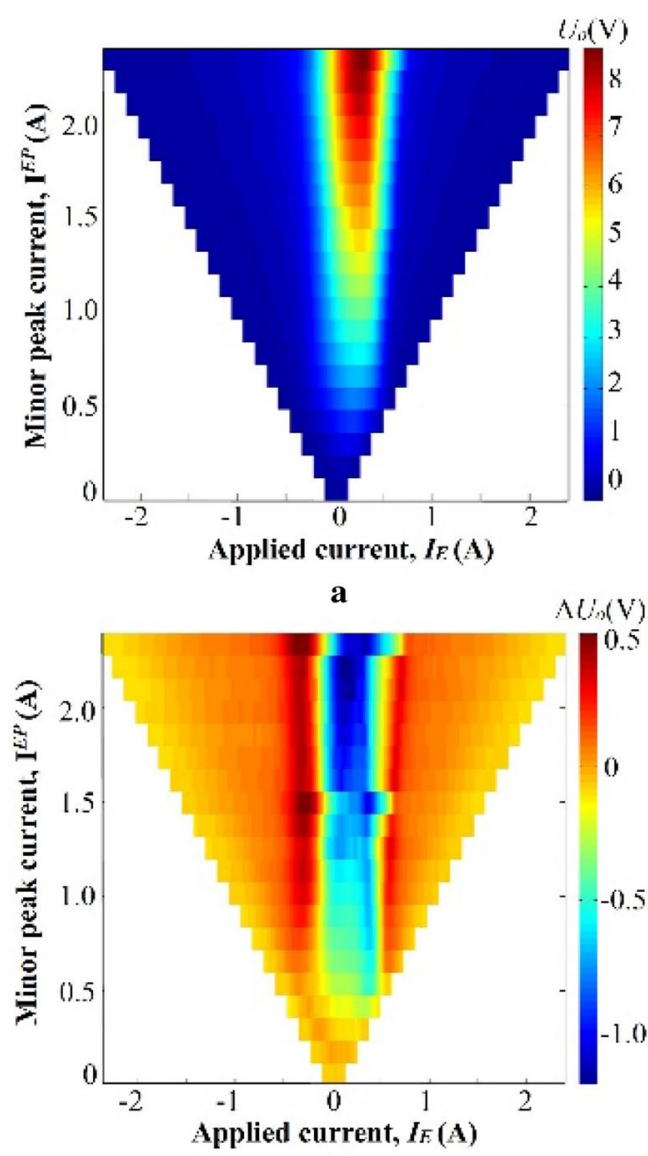

c

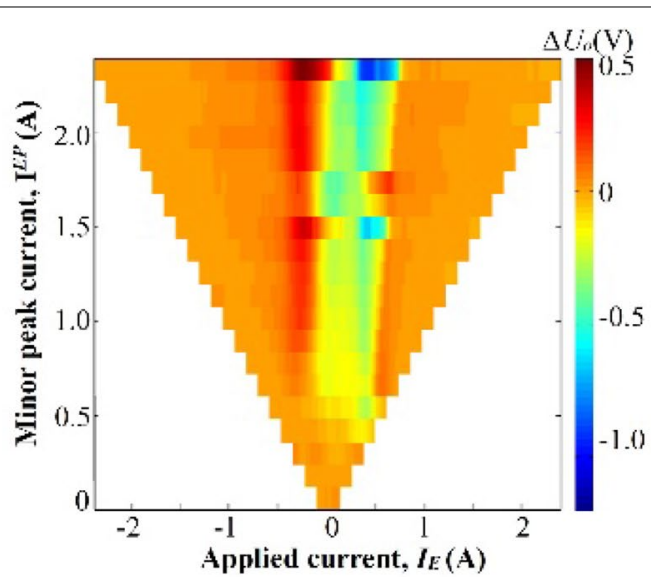

b

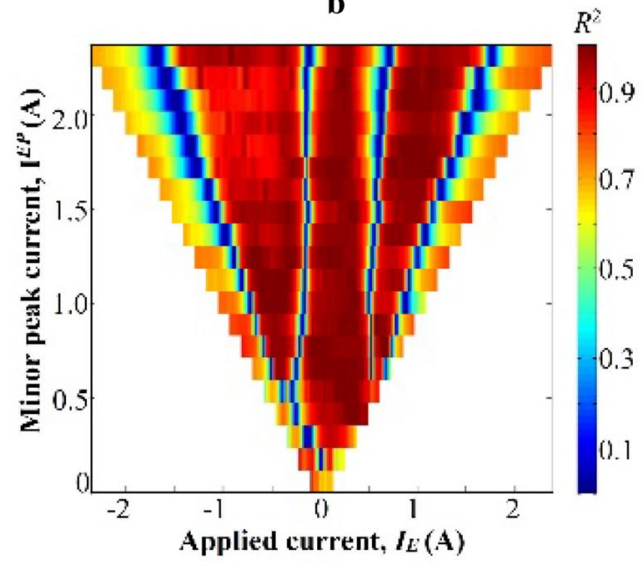

d

Figure 7 a $U_{0}$ matrix under the tensile force of $0 \mathrm{kN}$; To highlight the tensile force-induced voltage variation in the matrix, the $U_{0}$ matrix shown in $\mathbf{a}$ is subtracted from the matrix for the cases of $F=39.2 \mathrm{kN}$ and $F=78.4 \mathrm{kN}$ and the obtained results are shown in $\mathbf{b}$ and $\mathbf{c}$, respectively; Linear curve fitting operation is applied to the dataset of induced voltages and the tensile forces and the $R^{2}$ map is shown in $\mathbf{d}$

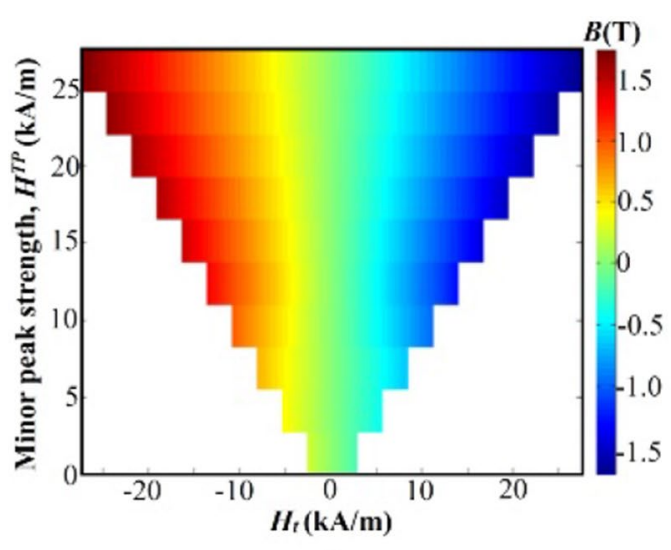

$\mathbf{a}$

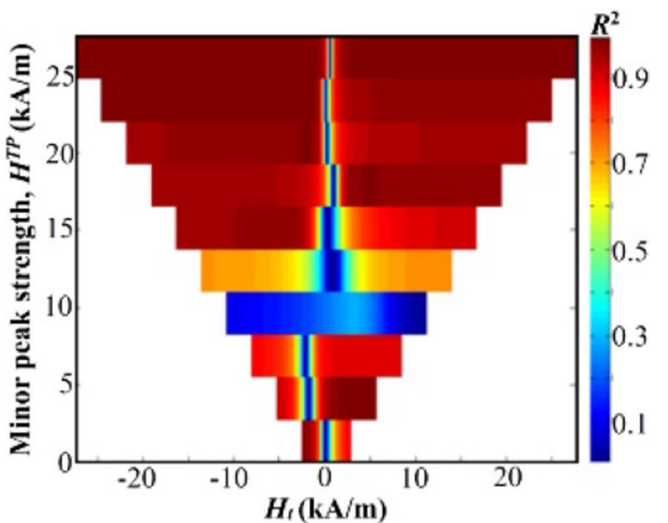

b

Figure $\mathbf{8}$ a $B$ matrix obtained from the raw material without induction-hardened layer, and $\mathbf{b} R^{2}$ matrix for evaluating the degree of linear correlation between the magnetic induction intensity and the effective case depth 
peak strength $\boldsymbol{H}^{T P}$ (referred as the peak amplitude of each cycle of the applied magnetic field) is around $10 \mathrm{kA} / \mathrm{m}$. A narrow region near the middle line of the isosceles triangular map has the small value of $R^{2}$ (around 0.1), indicating that the linear correlation is not suitable to illustrate the relationship between the magnetic induction intensity and the effective case depth. Only the pixels with a value of $R^{2}$ larger than 0.8 are concerned since the linear dependency of the magnetic induction intensity on the effective case depth is expected.

The final results of the sensitivity map for the two studied cases are shown in Figure 9. Sensitivity nulling operation is applied to the pixels with a corresponding $R^{2}$ lower than 0.8 . A positive sensitivity indicates that the value of the selected minor-loop parameters demonstrates the approximately linear growth trend with the increase in the value of target properties. For the regions with negative sensitivity, the increase in tensile force (or case depth) will result in the linear decrease in the induced voltage (or magnetic induction intensity). Inside the triangle area of the sensitivity map, the sensitivity value of the pixels with a corresponding $R^{2}$ lower than 0.8 is set to be null. The pixels with a null sensitivity are shown as white pixels in the map.

In Figure 9a, the pixels with the high sensitivity are mainly concentrated in the region around the zero-current line, indicating that the material is in its remanence point at each cycle of minor loop. In the near-saturation magnetization region, the sensitivity is relatively lower than that obtained around the remanence point. When the amplitude of the excitation current is fixed, the sensitivity depends on the excitation cycle (with a minor peak current $I^{E P}$ ) or the magnetization degree of the material. The highest absolute value of the sensitivity is achieved when the excitation current is around $0.22 \mathrm{~A}$ at the last excitation cycle (with the highest $I^{E P}$ ).

Under the condition of the applied current corresponding to the highest sensitivity, it is found that the induced voltage in the secondary coil reaches its maxima. As emphasized by Faraday law of electromagnetic induction, the induced voltage is proportional to the change rate of magnetic induction intensity over time, $\mathrm{d} B / \mathrm{d} t$. Uniform interpolation of the applied magnetic field leads to a constant rate of change of $H(t)$. Finally, the induced voltage becomes proportional to the calculated differential permeability of the material according to the following derivation,

$$
U_{o} \propto \frac{\mathrm{d} B}{\mathrm{~d} H} \cdot \frac{\mathrm{d} H}{\mathrm{~d} t} \propto \mu_{\mathrm{d}}
$$

According to Eq. (4), the highest sensitivity indicates that the strand is magnetized to have the maximum differential permeability. Therefore, if the differential permeability calculated from the minor hysteresis loop is selected as the feature parameter for tensile force characterization, the highest sensitivity is expected to appear around the previously identified pixel (with the highest sensitivity) in the map in Figure 9a. To quantitatively analyze the applied-current-dependent sensitivity of $U_{o}$ to the tensile force of strand, the results of the slopes of the fitted lines (referred to as the sensitivity), which are obtained at the pixels with the current amplitudes of $-0.35 \mathrm{~A}$ and $0.22 \mathrm{~A}$, are plotted in Figure 10a.

For the two investigated applied current cases, the upward trend in the absolute value of sensitivity can be concluded. Compared to the case of $I_{E}=-0.35$ A, the performance of the induced voltage, which is corresponding to the case of $I_{E}=0.22 \mathrm{~A}$, for characterizing the

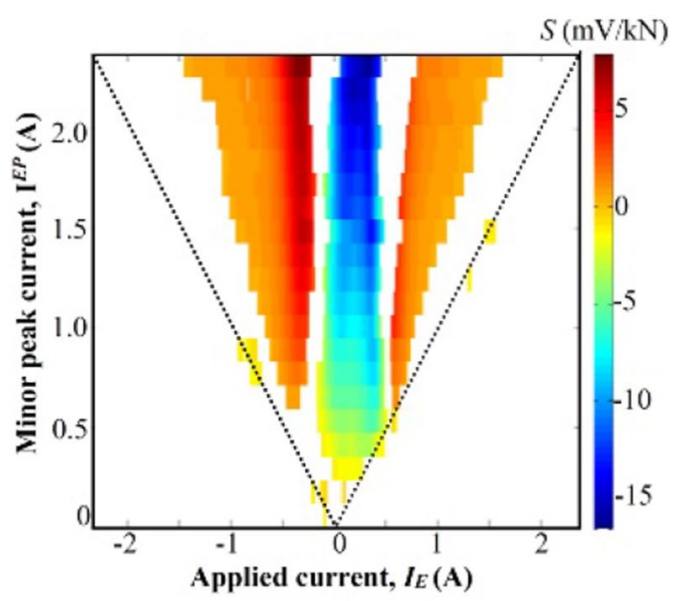

a

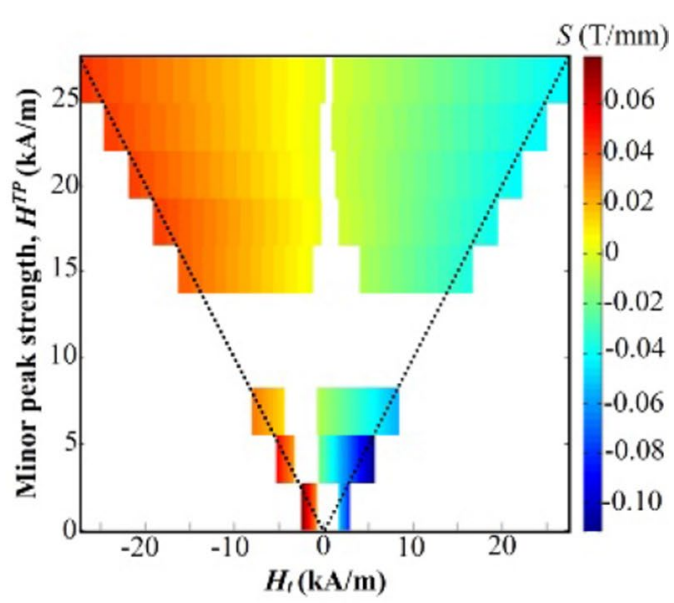

b

Figure 9 a Sensitivity map for the tensile force measurements in steel strand, and $\mathbf{b}$ Case depth evaluation in induction-hardened steel rod 


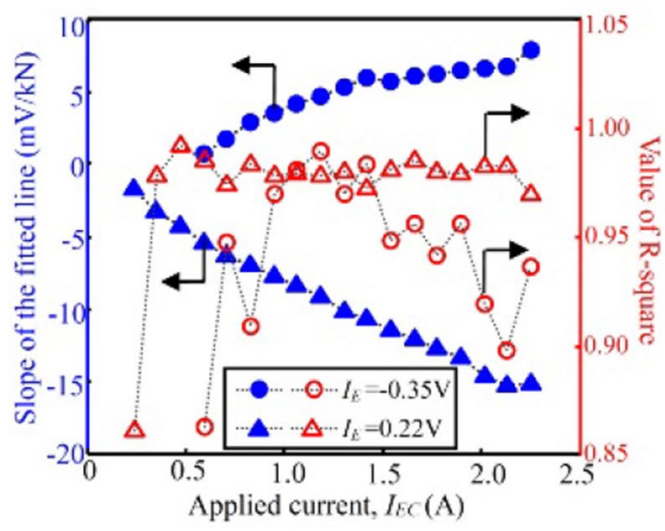

a

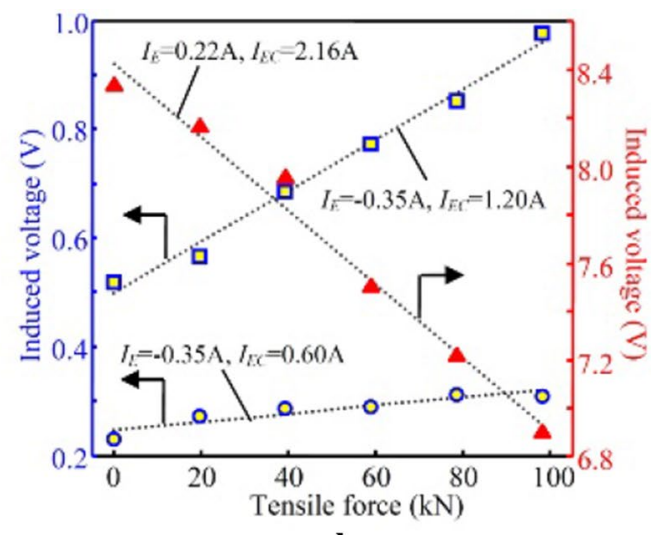

b

Figure 10 a Slope and R-square of the fitted lines for different columns in the matrix, and $\mathbf{b}$ Dependency of the induced voltage on the tensile force corresponding to different locations in the matrix

tensile force is much better due to the higher value of $R^{2}$ and absolute sensitivity in the majority of the range of $I^{E P}$. When the applied current is $I_{E}=-0.35 \mathrm{~A}$, the fitted line possessing the highest value of $R^{2}$ or absolute sensitivity together with the measured data is recalled and plotted in Figure 10b. The results obtained under the condition of $I_{E}=0.22 \mathrm{~A}$ and $I^{E P}=2.16 \mathrm{~A}$ are also plotted in Figure 10b for comparison. For the pixel with $I_{E}=-0.35 \mathrm{~A}$ and $I^{E P}=1.20 \mathrm{~A}$, the sensitivity of $U_{o}$ to the tensile force is around $4.71 \mathrm{mV} / \mathrm{kN}$, which can be enlarged by more than three times to a value of $15.26 \mathrm{mV} / \mathrm{kN}$ when the applied current of the pixel comes to $I_{E}=0.22 \mathrm{~A}$ and
$I^{E P}=2.16$ A. Therefore, sinusoidal current with as large peak-to-peak amplitude as possible is recommended as the excitation signal in the experiment. Consequently, the maximum strength of the applied magnetic field is strong enough to allow the strand to approach the saturation magnetization state. In this way, the high sensitivity of induced voltage to the tensile force can be achieved in the region where the change rate of magnetic induction intensity over time reaches its maxima.

The data of three rows with the minor peak strength of $H^{T P}=5.5 \mathrm{kA} / \mathrm{m}, H^{T P}=16.5 \mathrm{kA} / \mathrm{m}$ and $H^{T P}=27.5 \mathrm{kA} / \mathrm{m}$ in the sensitivity matrix are plotted in Figure 11. In the

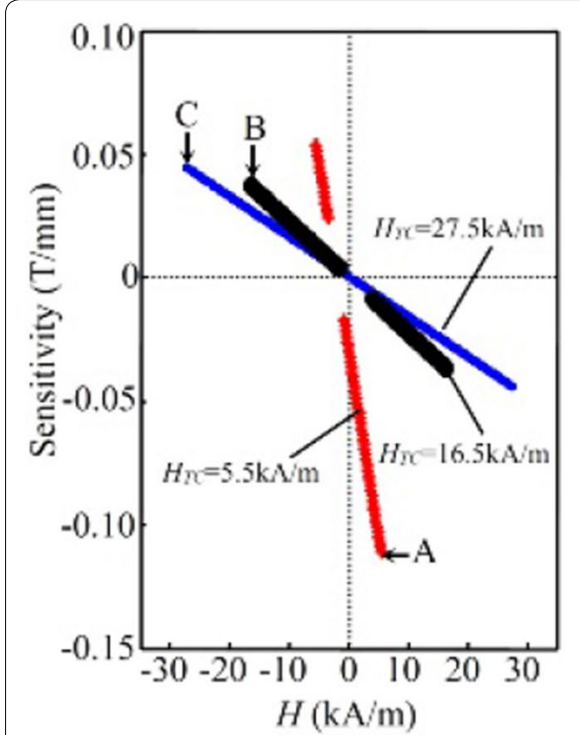

a
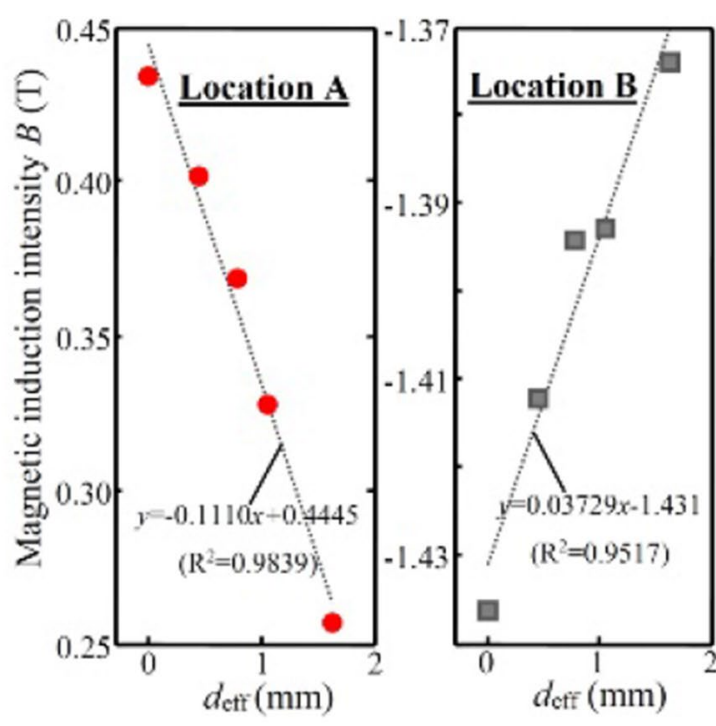

b

Figure 11 a Sensitivity at different locations in the matrix, and $\mathbf{b}$ Dependency of the magnetic induction intensity on the case depth at the locations of $A, B$ and $C$ marked in a 
investigated range of $H$, the linear dependency of the sensitivity on the magnetic field strength $H$ can be concluded. The sign of the sensitivity changes from positive to negative when the value of $H$ is around zero, which corresponds to the remanent magnetization state. Hence, unlike the case for tensile force measurement (Figure 9a), the sensitivity near the remanent magnetization region is extremely low. The asymmetric distribution of the absolute values of sensitivity in the negative and positive spaces of $H$ can be observed under the weak magnetization state $\left(H^{T P}=5.5 \mathrm{kA} / \mathrm{m}\right)$. When the value of $H^{T P}$ increases to $16.5 \mathrm{kA} / \mathrm{m}$ and $27.5 \mathrm{kA} / \mathrm{m}$, the lines of sensitivity becomes nearly symmetric about the origin in Figure 11a.

In the space of positive $H$, with the increase in the value of $H^{T P}$, the absolute value of sensitivity decreases, indicating that the material is getting closer to the saturation magnetization. The highest absolute value of the sensitivity is obtained at Point A, as marked in Figure 11a. Therefore, in the weak magnetization state, the magnetic induction intensity of the material is more sensitive to the variation of case depth than that in near-saturation regions. The measured data of $B$ and $d_{\text {eff }}$ corresponding to the marked Points A, B and C are plotted in Figure 11b. At Point $\mathrm{A}$, the absolute value of sensitivity is about $0.1110 \mathrm{~T} / \mathrm{mm}$, which is higher than that obtained at Point B $(S=0.03729 \mathrm{~T} / \mathrm{mm})$ and Point C $(S=0.04510 \mathrm{~T} / \mathrm{mm})$.

Although the straight line well fits the data measured under the condition corresponding to Points $\mathrm{B}$ and $\mathrm{C}$, the increment of $B$ caused by the increase in the effective case depth from $0.78 \mathrm{~mm}$ to $1.05 \mathrm{~mm}$ is small, thus resulting in the poor sensitivity of the sensor in this particular range of $d_{\text {eff }}$ In contrast, at Point $\mathrm{A}$, the performance of the parameter of $B$ on characterizing the effective case depth is much better than that obtained at Points B and C. Based on the conclusions obtained from the results in Figure 11, it is inferred that weak magnetization is required for conducting high sensitivity measurement of case depth in induction-hardened steel rods with the minor-loop measurement technique. Therefore, according to the sensitivity map in Figure 9, the maximum strength of the applied field can be lowered to the proper range whilst the sensor possesses high sensitivity.

\section{Conclusions}

Magnetic minor hysteresis loop measurement and sensitivity mapping technique are applied in the measurements of tensile force in steel strand and effective case depth in induction-hardened steel rods for the first time. To achieve high-sensitivity and nondestructive measurement of target properties (tensile force and case depth), the excitation current and the analyzed locations in the minor loops are optimized based on the sensitivity map. In most of the locations in the minor loops, the amplitude of the induced voltage (or the magnetic induction intensity) demonstrates the approximately linear dependency on the tensile force (or effective case depth). However, in the sensitivity map, the location of the pixel with the highest sensitivity differs between the two studied cases. Since the location of the pixel reflects the magnetization state of the tested materials, the sensitivity map can act as an effective tool for the optimization of excitation magnetic field or excitation current in the primary coil.

In the case of tensile force measurement, along each minor loop, the locations with the high sensitivity are around the zero-current line in the map and the zero-current line corresponds to the remanent state of the strand during each minor loop cycle. When the induced voltage is analyzed around the zero-current line, its sensitivity to the tensile force can be enhanced by increasing the peak-to-peak amplitude of sinusoidal excitation current. Therefore, the higher extent of magnetization of the material indicates the higher sensitivity of the sensor. When the applied current is around $I_{E}=0.22 \mathrm{~A}$ and $I^{E P}=2.16 \mathrm{~A}$, the highest value of sensitivity is obtained as about $15.26 \mathrm{mV} / \mathrm{kN}$.

When the target property is changed from tensile force to effective case depth, the locations of the pixels corresponding to the highest sensitivity also shift. Under the action of the magnetic field with maximum peak-to-peak strength, the magnetic induction intensity along the measured minor loop does not demonstrate the highest sensitivity in the sensitivity map. In contrast, the highest sensitivity occurs under the condition of the low magnetic strength and the absolute value of sensitivity is about $0.1110 \mathrm{~T} / \mathrm{mm}$. Therefore, the induction-hardened steel rods do not need to be magnetized to the near-saturation state if the highest sensitivity of magnetic induction intensity to effective case depth is required.

\section{Acknowledgements \\ The authors sincerely thanks to Bin Wu of Beijing University of Technology for his critical discussion and reading during manuscript preparation.}

\section{Authors' Contributions}

$\mathrm{CH}$ and $\mathrm{XL}$ were in charge of the whole trial; $\mathrm{WD}$ and $\mathrm{XL}$ wrote the manuscript; WD, MY and RZ prepared and conducted the experiments. All authors read and approved the final manuscript.

\section{Authors' Information}

Cunfu He received his PhD degree in Engineering Mechanics from Tsinghua University, China, in 1996. Currently, he is a Professor at NDT\&E Laboratory, College of Mechanical Engineering and Applied Electronics Technology, Beijing University of Technology, China. His research interests are in the areas of structural health monitoring (SHM) technology and micromagnetic materials characterization technology, etc. He is a member of CICS, CSTAM and CMES.

Wenqiao Dou is currently working towards her Master's degree in Instrument Engineering at College of Mechanical Engineering and Applied Electronics 
Technology, Beijing University of Technology, China. Her research interests focus on the micro-magnetic material characterization.

Xiucheng Liu received his PhD in Mechanical Engineering from Beijing University of Technology, China, in 2013. Currently, he is a professor at NDT\&E Laboratory, Beijing University of Technology, China. His research interest includes the structural health monitoring (SHM) technology and micromagnetic materials characterization technology, etc.

Meng Yang obtained his Master degree in Instrument Engineering from College of Mechanical Engineering and Applied Electronics Technology, Beijing University of Technology, China. His research interests focus on the micro-magnetic material characterization.

Ruifang Zhang obtained her Master degree in Instrument Engineering from College of Mechanical Engineering and Applied Electronics Technology, Beijing University of Technology, China. Her research interests focus on the magnetic measurement of tensile force in steel strands.

\section{Funding}

Supported by National Key R\&D Program of China (Grant No. 2018YFF01012300) and National Natural Science Foundation of China (Grant No. 11527801).

\section{Competing Interests}

The authors declare no competing financial interests.

Received: 2 August 2019 Revised: 4 November 2020 Accepted: 11 November 2020

Published online: 25 November 2020

\section{References}

[1] Z Ma, Y Ma, F Zheng, et al. Modeling of hysteresis loop and its applications in ferroelectric materials. Ceramics International, 2018, 44(4): 4338-4343.

[2] C Li, G Shu, B Xu, et al. Effects of neutron irradiation on magnetic properties of reactor pressure vessel steel. Nuclear Engineering \& Design, 2019 342: 128-132.

[3] H L Ding, R Gao, T Zhang, et al. Annealing effect on the microstructure and magnetic properties of 14\% Cr-ODS ferritic steel. Fusion Engineering and Design, 2015, 100: 371-377.

[4] S Kobayashi, F Gillemot, Á Horváth, et al. Investigation of effects of long-term thermal aging on magnetization process in low-alloy pressure vessel steels using first-order-reversal-curves. AIP Advances, 2017, 7(5): 056002 .

[5] G Vertesy, I Tomáš, S Kobayashi. Nondestructive evaluation of low carbon steel by magnetic adaptive testing. Nondestructive Testing and Evaluation, 2010, 25(2): 125-132.
[6] U Aydin, P Rasilo, F Martin, et al. Modelling the effect of multiaxial stress on magnetic hysteresis of electrical steel sheets: A comparison. IEEE Conference on Electromagnetic Field Computation (CEFC), Miami, USA, November13-16, 2016.

[7] O Perevertov, R Schäfer. Influence of applied tensile stress on the hysteresis curve and magnetic domain structure of grain-oriented Fe-3\% Si steel. Journal of Physics D: Applied Physics, 2014, 47(18): 185001.

[8] STakahashi, S Kobayashi, I Tomáš, et al. Comparison of magnetic nondestructive methods applied for inspection of steel degradation. NDT \& $E$ International, 2017, 91: 54-60.

[9] STakahashi, S Kobayashi, H Kikuchi, et al. Analysis of minor hysteresis loops of cold rolled low carbon steel. IEEE Transactions on Magnetics, 2006 42(11): 3782-3784.

[10] S Kobayashi, S Takahashi, Y Kamada, et al. A low-field scaling rule of minor hysteresis loops in plastically deformed steels. IEEE Transactions on Magnetics, 2010, 46(2): 191-194.

[11] S Kobayashi, T Murakami, Y Narita, et al. Effects of cold rolling on magnetic minor hysteresis loops of duplex stainless steels. Philosophical Magazine, 2017, 97(6): 419-430.

[12] I Tomáš, G Vértesy. Adaptive testing for nondestructive evaluation of degraded materials. Journal of Electrical Engineering, 2016, 67(4): 273-278.

[13] I Tomáš, G Vértesy, F Gillemot, et al. Nondestructive Magnetic Adaptive Testing of nuclear reactor pressure vessel steel degradation. Journal of Nuclear Materials, 2013, 432(1): 371-377.

[14] G Vértesy, I Tomáš. Complex characterization of degradation of ferromagnetic materials by magnetic adaptive testing. IEEE Transactions on Magnetics, 2013, 49(6): 2881-2885.

[15] Tino Gottschall, E Stern-Taulats, L Mañosa, et al. Reversibility of minor hysteresis loops in magnetocaloric Heusler alloys. Applied Physics Letters, 2017, 110(22): 223904.

[16] M H Yoon, Y Y Choi, J P Hon. Improvement in thrust force estimation of solenoid valve considering minor hysteresis loop. AlP Advances, 2016, 7(5): 056607.

[17] M A Zambrello, A D Schuyler, M W Maciejewski, et al. Nonuniform sampling in multidimensional NMR for improving spectral sensitivity. Methods, 2018, 138-139: 62-68.

[18] X C Liu, B Wu, F Qin, et al. Observation of ultrasonic guided wave propagation behaviours in pre-stressed multi-wire structures. Ultrasonics, 2017, 73: 196-205.

[19] X Yan, D Zhang, F Zhao. Improve the signal to noise ratio and installation convenience of the inductive coil for wire rope nondestructive testing. NDT \& E International, 2017, 92: 221-227.

[20] C Carlo, Z Daniele, L H Ait, et al. Calibration of elasto-magnetic sensors on in-service cable-stayed bridges for stress monitoring. Sensors, 2018, 18(2): 466.

[21] C F He, M Yang, X C Liu, et al. Characterisation of case depth in inductionhardened medium carbon steels based on magnetic minor hysteresis loop measurement technique. Philosophical Magazine, 2017: 1-16.

\section{Submit your manuscript to a SpringerOpen ${ }^{\circ}$ journal and benefit from:}

- Convenient online submission

- Rigorous peer review

- Open access: articles freely available online

- High visibility within the field

- Retaining the copyright to your article

Submit your next manuscript at springeropen.com 